\title{
Influence of fermentable carbohydrates or protein on large intestinal and urinary metabolomic profiles in piglets ${ }^{1}$
}

\author{
R. Pieper,* K. Neumann,† S. Kröger,* J. F. Richter,† J. Wang,§ L. Martin,* J. Bindelle,\# \\ J. K. Htoo, || V. Vahjen,* A. G. Van Kessel,§ and J. Zentek*2
}

\begin{abstract}
*Institute of Animal Nutrition, Department of Veterinary Medicine, Freie Universität Berlin, D-14195 Berlin, Germany; $\dagger$ †epartment of Biometry and Clinical Epidemiology, Charité Universitätsmedizin Berlin, D-10098, Germany; †Institute of Clinical Physiology, Charité Universitätsmedizin Berlin, D-12203 Berlin, Germany; §Department of Animal and Poultry Science, University of Saskatchewan, Saskatoon, Saskatchewan S7N 5A8, Canada; \#University of Liège, Gembloux AgroBio Tech, Animal Science Unit, 5030 Gembloux, Belgium; and \|Evonik Industries, D-63457 Hanau-Wolfgang, Germany
\end{abstract}

ABSTRACT: It was recently shown that variations in the ratio of dietary fermentable carbohydrates (fCHO) and fermentable protein (fCP) differentially affect large intestinal microbial ecology and the mucosal response. Here we investigated the use of mass spectrometry to profile changes in metabolite composition in colon and urine associated with variation in dietary fCHO and fCP composition and mucosal physiology. Thirty-two weaned pigletswere fed 4 diets in a $2 \times 2$ factorial design with low fCP and low fCHO, low fCP and high fCHO, high fCP and low fCHO, and high fCP and high fCHO. After 21 to $23 \mathrm{~d}$, all pigs were euthanized and colon digesta and urine metabolite profiles were obtained by mass spectrometry. Analysis of mass spectra by partial least squares approach indicated a clustering of both colonic and urinary profiles for each pig by feeding group. Metabolite identification and annotation using the Kyoto Encyclopedia of Genes and Genomes (KEGG) metabolic pathways revealed increased abundance of metabolites associated with arachidonic acid metabolism in colon of pigs fed a high concentration of fCP irrespective of dietary fCHO. Urinary metabolites did not show as clear patterns. Mass spectrometry can effectively differentiate metabolite profiles in colon contents and urine associated with changes in dietary composition. Whether metabolite profiling is an effective tool to identify specific metabolites (biomarkers) or metabolite profiles associated with gut function and integrity needs further elucidation.

Key words: colon, fermentable protein, fermentable fiber, metabolomics, pigs

(C) 2012 American Society of Animal Science. All rights reserved.

J. Anim. Sci. 2012.90:34-36 doi: $10.2527 /$ jas 53918

\section{INTRODUCTION}

High levels of undigested fermentable protein reaching the colon of pigs are associated with the formation of potentially harmful metabolites and impairment of intestinal barrier function. Balancing the ratio of fermentable protein to carbohydrates in the pig diet can reduce the level of potentially harmful colonic metabolites (Bikker et al., 2006). However, it was recently shown that, although fermentable carbohydrates (fCHO) can partly reverse fermentable

${ }^{1}$ Evonik Industries (Germany), the German Research Foundation (DFG) through grant \# SFB852/1, and the Canadian Swine Research and Development Cluster provided funding for this study. The Integrated Graduate School 'Biology of Nutrition' of the Collaborative Research Group SFB852 provided a doctoral stipend to J. Wang.

${ }^{2}$ Corresponding author: zentek.juergen@vetmed.fu-berlin.de protein (fCP)-associated changes in large intestinal microbial ecology, no effect on fCP-associated changes in gut parameters such as mucosal cytokine gene expression and oxidative stress response were identified (Pieper et al., 2012).

Traditionally, short and branched chain fatty acids, $\mathrm{NH}_{3}$, and amines have been monitored in the porcine intestinal tract to draw conclusions on intestinal health. High throughput metabolite identification and quantification technologies such as mass spectrometry have made it possible to simultaneously assess thousands of metabolites in the intestinal ecosystem and the host. A challenge with the high resolution metabolomic approaches is data management. Recently, bioinformatic tools became available to analyze large datasets from metabolomic data, thus facilitating data handling and possible identification of biomarkers for 
gut health. This experiment was performed to assess high resolution mass spectrometry as a tool to investigate metabolite composition and identify potential biomarkers in colon contents and urine associated with changes in dietary composition and mucosal physiology.

\section{MATERIALS AND METHODS}

All procedures involving animal handling and treatments were approved by the local state office of occupational health and technical safety (Landesamt für Gesundheit und Soziales, Berlin, Regulation no. 0249/10). Thirty-two piglets (Euroc $\times$ Piétrain) were assigned to 1 of 4 treatments in a $2 \times 2$ factorial design with fCP and low fCHO [14.5\% CP and $4.5 \%$ total dietary fiber (TDF)], low fCP and high fCHO (14.8\% $\mathrm{CP}$ and $16.6 \%$ TDF), high fCP and low fCHO (19.8\% $\mathrm{CP}$ and $14.5 \% \mathrm{TDF}$ ), and high fCP and high fCHO (20.1\% CP and $18.0 \%$ TDF) as dietary treatments as specified by Pieper et al. (2012). To increase the amount of fermentable protein, soybean (Glycine max) meal was heat treated by steam autoclaving at $124^{\circ} \mathrm{C}$ for 20 min. Fermentable carbohydrates were supplied as wheat (Triticum aestivum) bran and sugar beet pulp to replace corn (Zea mays) and wheat. Pigs ( $\mathrm{n}=8$ per group) were euthanized each day from 20 to $23 \mathrm{~d}$ postweaning and digesta samples were taken from proximal colon. Urine was collected directly from the bladder.

For determinations of metabolite profiles, samples were acidified with $1.0 \%$ formic acid and deproteinated and desalinated using solid phase extraction on HyperSep C18 columns (Thermo Scientific). Finally the columns were washed with $0.1 \%$ formic acid and samples were eluted with $2 \mathrm{~mL}$ methanol. Mass spectra were obtained on an Agilent 6210 Electrospray-Ionization-Time-Of-Flight (ESI-TOF) mass spectrometer (Agilent Technologies, Santa Clara, CA) in full scan mode from 100 to 1000 $\mathrm{m} / \mathrm{z}$ in positive electrospray-ionization (ESI) mode. The injection flow rate was $10 \mu \mathrm{L} / \mathrm{min}$, the capillary voltage was $3500 \mathrm{~V}$, and the fragmentor was set at $250 \mathrm{~V}$. Data were obtained as .txt files and aligned using OpenMS (http:// open-ms.sourceforge.net/openms/). Partial least squares (PLS) analysis was performed using the caret package (version 4.91) of the R 2.13.0 software (R Foundation for Statistical Computing, Vienna, Austria) in order to obtain a list of unique metabolites for each feeding group, that is, metabolites that are differently expressed in this group. In particular for each metabolite the variable importance score was calculated. In order to validate PLS analysis, a permutation test was performed with 10,000 random permutations of the group assignments. A total of 200 discriminating putative metabolites $(\mathrm{m} / \mathrm{z}$ values) from colon contents and approximately 100 from urine samples were selected and submitted for identification and annotation into the Kyoto Encyclopedia of Genes and Genomes (KEGG) pathways using the MassTRIX software (http://metabolomics.helmholtz-muenchen.de/ masstrix2/; Suhre and Schmitt-Kopplin, 2008).

\section{RESULTS AND DISCUSSION}

Colonic contents and urine collected in this study were used to investigate the suitability of mass spectrometry to identify changes in metabolite composition associated with different diets. Results on animal performance, nutrient digestibility, intestinal microbial ecology, tissue gene expression, and oxidative stress were previously reported (Pieper et al., 2012). In brief, the study found increased expression of cell turnover, secreted mucin, and inflammatory genes in colonic mucosa associated with diets containing high fCP. Dietary inclusion of fCHO reduced for the abundance of some clostridia, amines, and $\mathrm{NH}_{3}$ in the colon associated with high fCP but did not affect the mucosal response (Pieper et al., 2012).

Analysis of metabolomic profiles from colon contents and urine revealed a distinct clustering of profiles for each pig associated with diet composition (Figure 1A and 1B). This shows that both intestinal contents and urine might serve as possible media to detect metabolites associated with different nutritional strategies. Because urine is more readily assessable than colon contents, urine may be the preferred medium; however, given the differences in clustering patterns both sampling sites may offer important associations. The identification of metabolites based on $\mathrm{m} / \mathrm{z}$ values and annotation into KEGG pathways revealed that group discriminating metabolites in the colon were associated with arachidonic acid, glycerophospholipid, and lysine degradation pathways (Figure 2A). We observed an increased abundance of metabolites associated with arachidonic acid metabolism including prostaglandin $\mathrm{E}_{2}$ and thromboxane $\mathrm{A}_{2}$ in groups receiving high concentration of fCP. This might reflect the increased pro- and anti-inflammatory cytokine
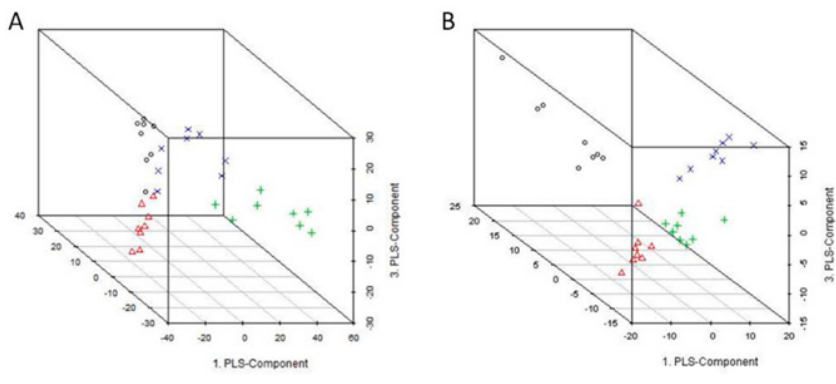

Figure 1. Scatter plot of partial least square (PLS) analysis of colon content (A) and urine (B) metabolite profiles showing the first three PLS components. Pigs were fed diets containing low fermentable protein (fCP) and low fermentable carbohydrates (fCHO, circle), low fCP and high fCHO (triangle), high $\mathrm{fCP}$ and low fCHO (plus sign), and high $\mathrm{fCP}$ and high fCHO $(\times)$, respectively. Each symbol represents the metabolite profile of an individual pig. 

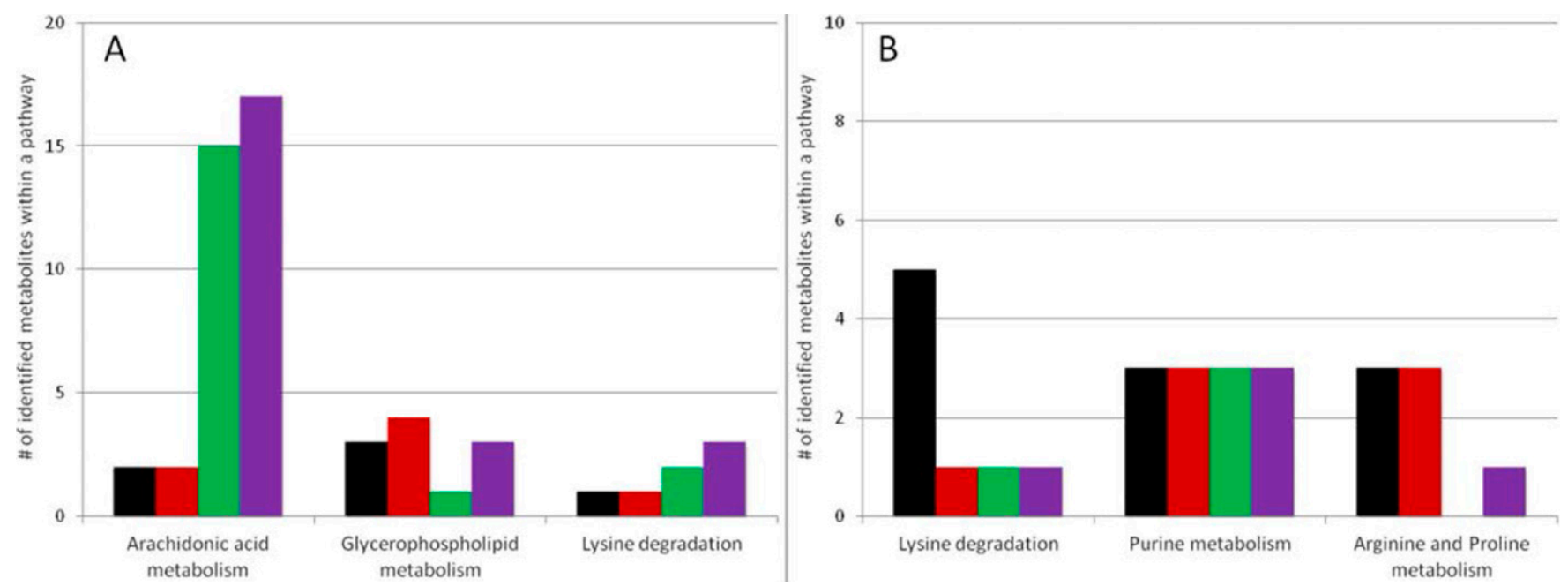

Figure 2. Identified metabolites from colon content (A) and urine (B) samples within the (KEGG) pathways that discriminated the four groups after partial least square (PLS) analysis and variable importance (VIP) scoring. Pigs were fed diets containing low fermentable protein (fCP) and low fermentable

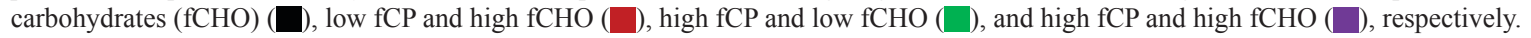

expression in the high fCP supplemented groups as reported previously (Pieper et al., 2012). Prostaglandins including prostaglandin $\mathrm{E}_{2}$ act as signaling molecules and are involved in recovery of intestinal tissue barrier function (Blikslager et al., 1999). Interestingly, an increased abundance of metabolites associated with lysine degradation was observed in colon contents of pigs fed high fCP diets whereas this picture was reversed in urine samples (Figure 2B). Reasons are not yet clear but could reflect the increased flow of undigested amino acids from heat treated soybean meal in high fCP groups and different metabolism of amino acids in low fCP supplemented pigs. However, the number of identified metabolites from urine samples was lower compared with colon samples. Finally, the identification of arachidonic acid and lysine degradation metabolites in colon contents underlines that this medium contains both host- and microbial-origin metabolites, which contribute to profiles associated with changes in diet composition.

In conclusion, high resolution mass spectrometry in combination with bioinformatic tools can differentiate metabolite profiles in colon contents and in urine associated with changes in diet composition and host physiology. The methodology may be valuable to identify unique biomarkers associated with gut health.

\section{LITERATURE CITED}

Bikker, P., A. Dirkzwager, J. Fledderus, P. Trevisi, I. le Huerou-Luron, J. P. Lalles, and A. Awati. 2006. The effect of dietary protein and fermentable carbohydrates levels on growth performance and intestinal characteristics in newly weaned piglets. J. Anim. Sci. 84:3337-3345.

Blikslager, A. T., M. C. Roberts, and R. A. Argenzio. 1999. Prostaglandin-induced recovery of barrier function in porcine ileum is triggered by chloride secretion. Am. J. Physiol. Gastrointest. Liver Physiol. 276:G28-G36.

Pieper, R., S. Köger, J. F. Richter, J. Wang, L. Martin, J. Bindelle, J. K. Htoo, D. von Smolinski, W. Vahjen, J. Zentek, and A. G. Van Kessel. 2012. Fermentable fiber ameliorates fermentable protein-induced changes in microbial ecology, but not the mucosal response, in the colon of piglets. J. Nutr. 142:661-667.

Suhre, K., and P. Schmitt-Kopplin. 2008. MassTRIX: Mass translator into pathways. Nucleic Acids Res. 36:W481-W484. 